jacent views. Similarly, the horizontal resolution along the line of sight of a satellite sensor can be improved by observing along the satellite track, and oversampling. Some of these improvements are included in the satellite radiometer HIRDLS (ref. 4).

Vertical resolution could also be improved by adding filters which select radiation from parts of the spectrum with weak lines, by choosing longer wavelengths where possible, and by measuring source gases. As the spectral bands of $\mathrm{N}_{2} \mathrm{O}$ and $\mathrm{CH}_{4}$ interfere in the mid-infrared, a radiometer that could measure a linear combination of $\mathrm{N}_{2} \mathrm{O}$ and $\mathrm{CH}_{4}$ would be simpler than one that measured either constituent separately, but would still be suitable for identifying exvortex air. A radiometer observing a chlorofluorocarbon at $11-12 \mu \mathrm{m}$ would have the advantages of longer wavelength and weaker lines, but with less signal.

A balloon-borne emission sensor could achieve much better resolution by using the viewing geometry shown in the figure, not possible from satellites. By taking the difference between signals $0.6^{\circ}$ below and $0.6^{\circ}$ above the horizontal, with a field of view of less than $0.2^{\circ}$, the resolution would be better than 0.5 by $150 \mathrm{~km}$. The required pointing accuracy of $0.05^{\circ}$ is easily achieved by modern angle encoders and gyroscopes. At the expense of signal, the resolution could be improved further by reducing the elevation and depression of the viewing angles, provided that the field of view and pointing error were reduced accordingly. The measurement assumes that the atmosphere is horizontally uniform for $150 \mathrm{~km}$ - provided the signalto-noise ratio were sufficiently high, this assumption could be tested by allowing the instrument to rotate freely and by comparing observations in each quadrant.

Modern limb-sounders have programmable viewing, so that the geometry of the figure could be used during ascent and a valved descent, while retaining the conventional limb scan and floating at constant altitude. Not only would this enhance the capability of general balloon-borne remote sensing, but the geometry of the figure could also be used by high-altitude aircraft provided the viewing system were interfaced to the aircraft's inertial platform.

Unfortunately, there seem to be no plans to implement the simple balloon-borne geometry of the figure, which could make observations at improved resolution and at low cost.

British Antarctic Survey,

H. K. Roscoe

Natural Environment Research Council, Madingley Road,

Cambridge CB3 OET, UK

1. Tuck, A.F., Proc. First Europ. Workshop Polar Strat. Ozone Res. Schliersee, 3-5 Oct. 1990 (in the press).

2. Toohey, D.W., Brune. W.H., Chan, K. R. \& Anderson, J. G. Geophys. Res. Lett. 18, 21-24 (1991).

3. WMO Global Ozone Research and Monitoring Project Rep. No. $20(1990)$

4. Barnett, J. J. et al. Oxford University proposal to NASA (1 July 1988).

\section{No capillary rise?}

$\mathrm{S}_{\mathrm{IR}}-$ The rise of liquid menisci in capillary tubes is always assumed to be described by the Kelvin equation'. But I have found that the menisci in test-tubes a few millimetres in diameter, immersed halfway into a sealed reservoir of water with the open end at the top, do not rise to the Kelvin level even after several years. Whereas the menisci in neighbouring open tubes do display the expected capillary rise, those for the half-closed tubes attain the same level as that of the reservoir, irrespective of tube diameter, initial level or temperature (for $30^{\circ} \mathrm{C}<T<55^{\circ} \mathrm{C}$ ). This behaviour is as though the surface tension of the water were zero, even though the menisci

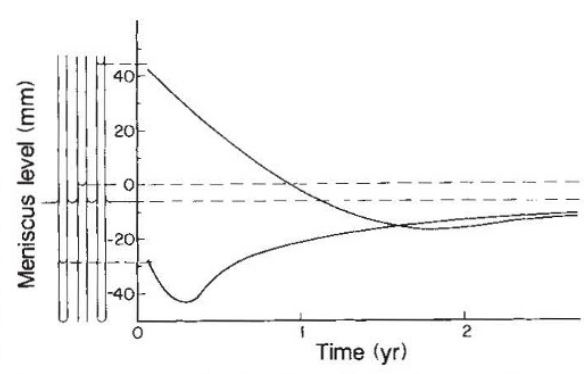

Meniscus levels in the half-closed 4-mm diameter tubes at room temperature.

in the half-closed tubes were as hemispherical as those in the open tubes.

Six 9 -cm-long pyrex tubes, three of 4-mm internal diameter and three of $1-\mathrm{mm}$ diameter, were mounted, in parallel, using pyrex spacers, inside a pyrex vessel $5 \mathrm{~cm}$ in diameter. One tube of each size was open at both ends, and the others were closed at one end. The system was cleaned with hydrofluoric acid, detergent solution, alcohol and distilled water. The vessel was half filled with triply distilled water, pumped to the freezing point and sealed off. By manipulating the orientation it was possible to fill one of the larger tubes and have the other nearly empty, leaving both smaller tubes approximately half full. This initial manipulation also had the effect of coating all walls in the system with the original sample of water. The vessel was placed in a room-temperature enclosure and the levels were monitored with an accuracy of $\pm 0.01 \mathrm{~mm}$.

After two years, the levels in all four closed tubes came to lie about $5 \mathrm{~mm}$ below the bulk reservoir level. The figure shows the results for the two 4-mm tubes. The vessel was then mounted in a thermostat set to $30{ }^{\circ} \mathrm{C}$. The levels changed at once, those in the 1-mm tubes rising four times faster than those in the 4-mm tubes; but all four levels rose only to the reservoir level.

Over the next 18 months, the temperature was raised in $8^{\circ} \mathrm{C}$ steps, held at each step for several months to a maximum of $55^{\circ} \mathrm{C}$, and then stepped back again to $30^{\circ} \mathrm{C}$, at which temperature it was held for a final eight months. The levels in the four closed tubes did not move by more than $0.05 \mathrm{~mm}$, remaining at the bulk meniscus level.
At the end of the experiment, mass spectrography showed only modest contamination of the water: boron and sodium were present at a few parts per million, as expected after the long exposure to pyre ${ }^{2}$. I found no systematic variation of impurity levels in samples from the reservoir or from the two sizes of half-closed tube. The following facts also argue against contamination effects; the internal consistency of the response to temperature changes (all four levels changed in the same way), the full hemispherical form of the menisci and the full capillary rise displayed in the open tubes, and the independence of the final menisci levels on the initial amount of water in the tubes. The last point was checked by observing initially full and nearly empty tubes of the same radius. When these had come to the same final level, the former would have concentrated its original contaminants threefold, whereas the latter would have diluted them by half. Thus a difference of a factor of six in contaminant concentrations made no observable difference in meniscus level. This is the strongest argument against such subtle effects as the migration of minute amounts of hydrophobic impurities to the menisci. Volume-distributed contamination would anyway be expected to raise the levels in half-closed tubes above the Kelvin level, rather than the reverse, because of osmotic pressure. This is also the expected effect of fluctuations of the ambient temperature acting through the nonlinear response of the vapour pressure.

Classically, the meniscus approaches the equilibrium level exponentially, driven by an evaporation-condensation imbalance with a relaxation time of the order of one month. However, this depends on the near equality of two very large effects which may be modified differently by the presence of nearby walls. Recently, direct observation of a metastable dry state on walls only a few millimetres above a normal meniscus has been reported $^{3}$. I suggest that metastable drying on the walls might be responsible for depression of the levels in the half-closed tubes in this experiment. (These experiments were performed at the University of Toronto.)

Physics Department,

University of California,

Irvine, California 92713, USA

1. Thomson, W. T. Phil. Mag 42, 448 (1871).

2. Adams, P. B. in Ultra Purity. Methods and Techniques (eds Zief, M. \& Speights, R.) 293-351 (Dekker, New York, 1972).

3. Garoff, S., Sirota, E. B., Sinha, S. K. \& Stanley, H. B. J. chem Phys. 90, 7505-7515 (1989).

\section{Scientific Correspondence}

Scientific Correspondence is intended to provide a forum in which readers may raise points of a scientific character. They need not arise out of anything published in Nature. In any case, priority will be given to letters of fewer than 500 words and five references.

NATURE $\cdot$ VOL $350 \cdot 21 \mathrm{MARCH} 1991$ 